University of South Carolina

Scholar Commons

\title{
Modification of Newman's BAND(J) Subroutine to Multi-Region Systems Containing Interior Boundaries: MBAND
}

D. Fan

Texas A \& M University - College Station

Ralph E. White

University of South Carolina - Columbia, white@cec.sc.edu

Follow this and additional works at: https://scholarcommons.sc.edu/eche_facpub

Part of the Chemical Engineering Commons

\section{Publication Info}

Journal of the Electrochemical Society, 1991, pages 1688-1691.

(c) The Electrochemical Society, Inc. 1991. All rights reserved. Except as provided under U.S. copyright law, this work may not be reproduced, resold, distributed, or modified without the express permission of The Electrochemical Society (ECS). The archival version of this work was published in the Journal of the Electrochemical Society.

http://www.electrochem.org/

DOI: $10.1149 / 1.2085854$

http://dx.doi.org/10.1149/1.2085854

This Article is brought to you by the Chemical Engineering, Department of at Scholar Commons. It has been accepted for inclusion in Faculty Publications by an authorized administrator of Scholar Commons. For more information, please contact digres@mailbox.sc.edu. 


\title{
Modification of Newman's BAND(J) Subroutine to Multi-Region Systems Containing Interior Boundaries: MBAND
}

\author{
D. Fan* and R. E. White ${ }^{* *}$ \\ Department of Chemical Engineering, Texas A\&M University, College Station, Texas 77843
}

\begin{abstract}
Newman's BAND(J) subroutine, which has been used widely to solve models of various electrochemical systems, is extended to solve a system of coupled, ordinary differential equations with interior boundary conditions. A set of coupled, linear ordinary differential equations is used to demonstrate the solution procedure. The results show that the extended technique has the same accuracy as that of using pentadiagonal BAND $(J)$, but the execution speed is about five times faster than that of pentadiagonal BAND(J). Using sparse matrix solver Y12MAF to solve the same set of equations takes even longer time than pentadiagonal BAND(J).
\end{abstract}

Electrochemical systems such as batteries and fuel cells consist of multiple regions as demonstrated through several references (1-4). The phenomena that occur in these regions can be modeled mathematically. This procedure yields a coupled set of equations with interior boundary conditions. Newman's BAND(J) subroutine $(5,6)$ has been used widely to solve systems of coupled, nonlinear ordinary differential equation. This is done by first transforming these sets of differential equations into sets of nonlinear algebraic equations by using finite difference approximations. These sets of nonlinear algebraic equations are then solved by using the Newton-Raphson procedure with BAND $(J)$. This procedure yields sets of linear of algebraic equations of the form $\mathbf{J C}=\mathbf{G}$ or

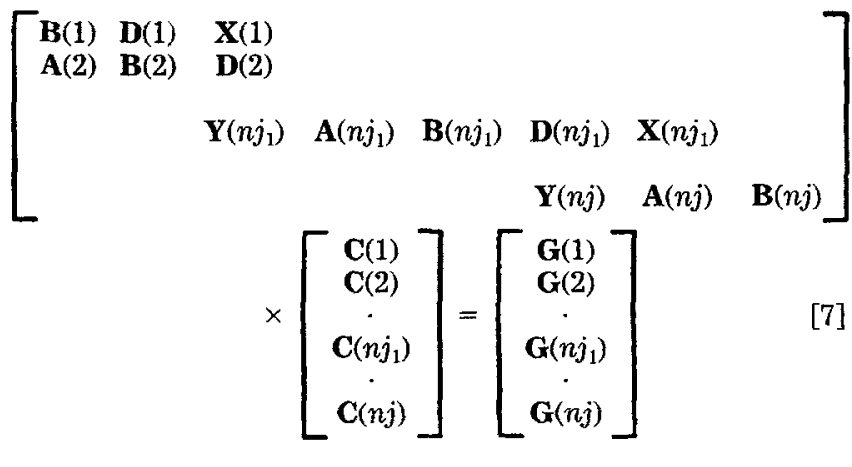

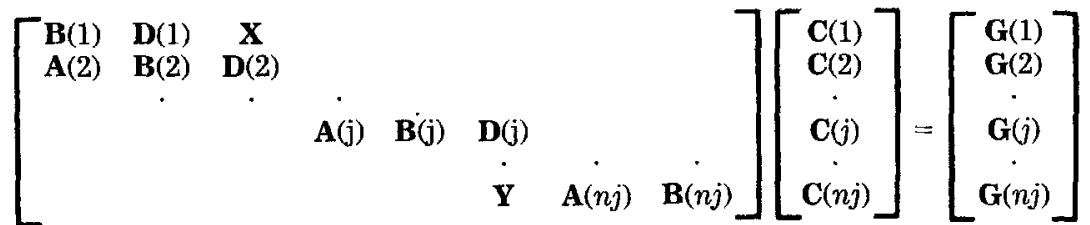

where Newman's notation (6) is used for convenience.

\section{Multiple Regions}

Most electrochemical systems include multiple regions (e.g., anode, separator, and cathode in a battery). Sets of differential equations with interior boundary conditions are used to model such devices. For example, in a tworegion system, there are two differential equations within their corresponding domain of $x$

$$
\begin{array}{lll}
F_{1}\left(x, \mathbf{c}, \mathbf{c}^{\prime}, \mathbf{c}^{\prime \prime}\right)=0 & \text { at } & 0<x<l_{1} \\
F_{2}\left(x, \mathbf{c}, \mathbf{c}^{\prime}, \mathbf{c}^{\prime \prime}\right)=0 & \text { at } & l_{1}<x<l_{2}
\end{array}
$$

where $\mathbf{c}=\left[c_{1}, c_{2}, \ldots, c_{n}\right]^{T}$ with boundary conditions of the form

$$
\begin{aligned}
& x=0: \alpha_{1} \mathbf{c}+\beta_{1} \frac{\partial \mathbf{c}}{\partial x}=\mathbf{f}_{1}(x) \\
& x=l_{1}:\left.\quad \alpha_{2} \frac{\partial \mathbf{c}}{\partial x}\right|_{-}=\left.\beta_{2} \frac{\partial \mathbf{c}}{\partial x}\right|_{+} \\
& x=l_{2}: \alpha_{3} \mathbf{c}+\beta_{3} \frac{\partial \mathbf{c}}{\partial x}=\mathbf{f}_{3}(x)
\end{aligned}
$$

Applying Newman's procedure (7) with three-point finite difference approximations used at all nodes including $x=l_{1}\left(j=n j_{1}\right)$ yields

\footnotetext{
* Electrochemical Society Student Member.
}

** Electrochemical Society Active Member. where the block jacobian coefficient matrix on the left side of Eq. [7] contains a row which has five elements instead of three (the row at $j=n j_{1}$ ). This is a result of using threepoint forward and backward finite difference formulas at the interface boundary to maintain accuracy to $O\left(h^{2}\right)$. The pentadiagonal BAND $(J)$ subroutine $(8)$ has been used $(9,1)$ to solve Eq. [7], but this is costly in terms of computation time because of the unnecessary computations due to the null matrices off the tridiagonal, especially when a large number of nodal points are used. However, it is important to note that this procedure is less costly than using forward and backward two-point finite difference approximations at the interface because such approximations are only accurate to $\mathrm{O}(h)$. It is possible to use other procedures

Table 1. Averaged relative error with respect to the exact solution using 21 nodes.

\begin{tabular}{lcc}
\hline Pentadiagonal BAND & Y12MAF & MBAND \\
\hline $4.973 \times 10^{-5}$ & $4.973 \times 10^{-5}$ & $4.973 \times 10^{-5}$
\end{tabular}

Table II. CPU time (ms) statistics for pentadiagonal BAND and MBAND.

\begin{tabular}{lcc}
\hline Module & Pentadiagonal BAND & MBAND \\
\hline MAIN & $25(6.94 \%)$ & $24(30.13 \%)$ \\
BAND & $290(82.19 \%)$ & $29(37.44 \%)$ \\
LUDEC & $27(7.51 \%)$ & $19(24.62 \%)$ \\
ZEROS & $12(3.35 \%)$ & $6(7.81 \%)$ \\
TOTAL & 353 & 78
\end{tabular}


(10) to maintain accuracy to $\mathrm{O}\left(h^{2}\right)$ at interior boundaries, but these may be difficult to apply especially to systems with several regions. A modified $B A N D(J)$, named MBAND, is presented here that reduces significantly the computation time to solve Eq. [7]. (The source code for this subroutine is available from the authors upon request.)

When LU decomposition is performed on the jacobian coefficient matrix in Eq. [7], the upper and lower triangular matrices that result are as follows $\mathbf{b}(1) \cdot[-\mathbf{x}]=\mathbf{X}$

where $\mathbf{E}(1)$ and $\mathbf{x}$ can be determined by solving $\mathbf{E q}$. [12] and [13] by knowing $\mathbf{b}(1)$ from Eq. [11]. For the second row of Eq. [8], the formulas are slightly different

$$
\mathbf{a}(2) \cdot \mathbf{I}=\mathbf{A}(2)
$$

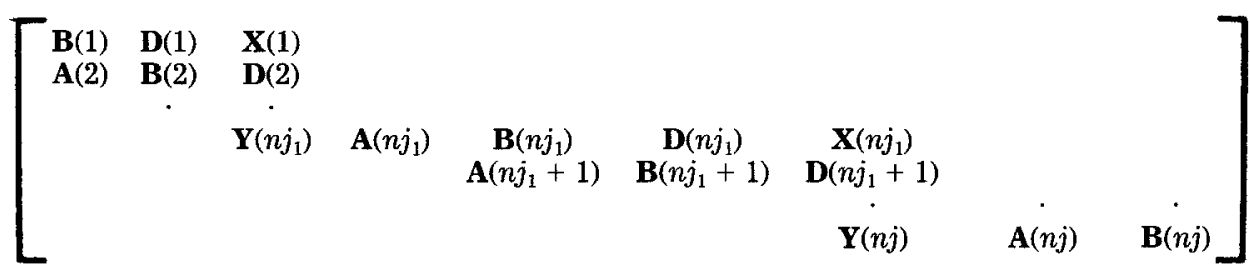

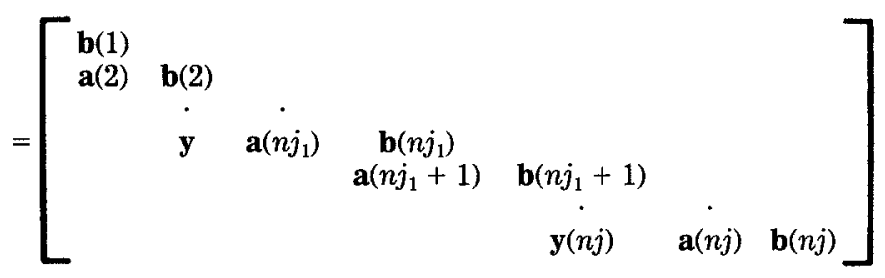

$$
\times\left[\begin{array}{cccccc}
\mathbf{I} & -\mathbf{E}(1) & -\mathbf{x}(1) & & & \\
& \mathbf{I} & -\mathbf{E}(2) & & & \\
& \cdot & \cdot & & \\
& & \mathbf{I} & -\mathbf{E}\left(n j_{1}\right) & -\mathbf{x}\left(n j_{1}\right) & \\
& & & \mathbf{I} & -\mathbf{E}\left(n j_{1}+1\right) & \\
& & & & & \mathbf{I}
\end{array}\right]
$$

The matrix on the right side of Eq. [8] can be used to solve

Eq. [7] by defining an intermediate vector $\mathbf{v}$ such that

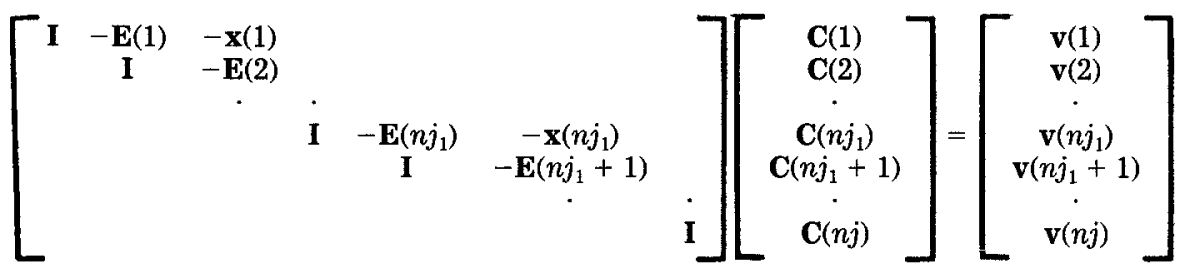

which yields

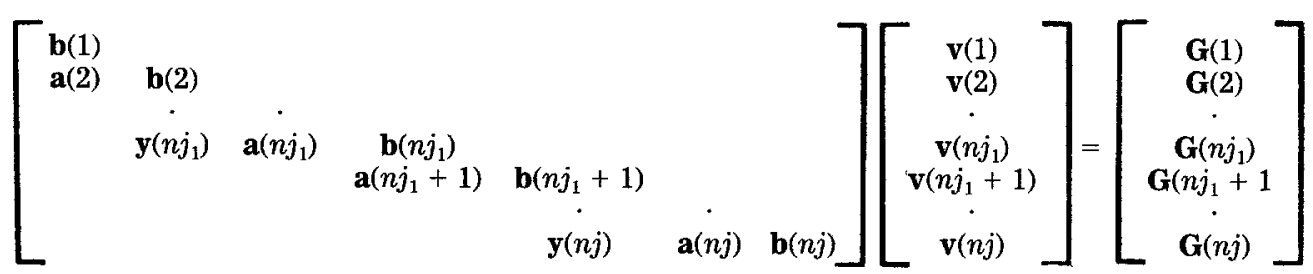

The coefficient matrices in Eq. [9] and [10] are obtained by solving Eq. [8] row by row. Solving the first row of Eq. [8] will give the values of elements in matrices $b(1)$, $\mathbf{E}(1)$, and $\mathbf{x}(1)$. These values are used to calculate the elements in the next row $\mathbf{a}(2), \mathbf{b}(2)$, and $\mathbf{E}(2)$. The process could be repeated until all of the elements on the right side of Eq. [8] are determined.

Next the intermediate vector $\mathbf{v}$ is obtained by using forward substitution to solve Eq. [10]. Once all of the $\mathbf{a}, \mathbf{b}$, and $\mathbf{E}$ matrices and the $\mathbf{v}$ vector are determined, the unknown vectors $\mathbf{c}(1), \ldots, \mathbf{c}(n j)$ can be obtained by using backward substitution to solve Eq. [9].

The equations obtained by these procedures are given next. For the first row of Eq. [8], we obtain the following matrix equations

$$
\begin{gathered}
\mathbf{b}(1) \cdot \mathbf{I}=\mathbf{B}(1) \\
\mathbf{b}(1) \cdot[-\mathbf{E}(1)]=\mathbf{D}(1)
\end{gathered}
$$

$$
\mathbf{a}(2) \cdot[-\mathbf{E}(1)]+\mathbf{b}(2) \cdot \mathbf{I}=\mathbf{B}(2)
$$

or

$$
\mathbf{b}(2)=\mathbf{B}(2)+\mathbf{a}(2) \cdot \mathbf{E}(1)
$$

In Eq. [16], since all matrices on the right side are known, b(2) can be calculated directly. This matrix can then be used to find values of $\mathbf{E ( 2 )}$ according to Eq. [8]

$$
\mathbf{a}(2) \cdot[-\mathbf{x}]+\mathbf{b}(2) \cdot[-\mathbf{E}(2)]=\mathbf{D}(2)
$$

or

$$
\mathbf{b}(2) \cdot[-\mathbf{E}(2)]=\mathbf{D}(2)+\mathbf{a}(2) \cdot \mathbf{x}
$$

and $\mathbf{v}(2)$ according to Eq. [10]

$$
\mathbf{a}(2) \cdot \mathbf{v}(1)+\mathbf{b}(2) \cdot \mathbf{v}(2)=\mathbf{G}(2)
$$


or

$$
\mathbf{b}(2) \cdot[-\mathbf{v}(2)]=-\mathbf{G}(2)+\mathbf{a}(2) \cdot \mathbf{v}(1)
$$

Equations [18] and [20] must be solved to get the values of $\mathbf{E}(2)$ and $\mathbf{v}(2)$.

A similar technique applies to the nodes at $j$ from 3 through $n j_{1}-1$, and from $n j_{1}+3$ through $n j-1$

$$
\mathbf{a}(j) \cdot \mathbf{I}=\mathbf{A}(j)
$$

and

$$
\mathbf{a}(j) \cdot[-\mathbf{E}(j-1)]+\mathbf{b}(j) \cdot \mathbf{I}=\mathbf{B}(j)
$$

or

$$
\mathbf{b}(j)=\mathbf{B}(j)+\mathbf{a}(j) \cdot \mathbf{E}(j-1)
$$

and

$$
\mathbf{b}(j) \cdot[-\mathbf{E}(j)]=\mathbf{D}(j)
$$

and according to Eq. [10]

$$
\mathbf{a}(j) \cdot \mathbf{v}(j-1)+\mathbf{b}(j) \cdot \mathbf{v}(j)=\mathbf{G}(j)
$$

or

$$
\mathbf{b}(j) \cdot[-\mathbf{v}(j)]=-\mathbf{G}(j)+\mathbf{a}(j) \cdot \mathbf{v}(j-1)
$$

where $\mathbf{b}(j)$ in Eq. [23] is directly calculated and then Eq. [24] and [26] are solved to obtain $\mathbf{E}(j)$ and $\mathbf{v}(j)$. The equations at the interior boundary $\left(j=n j_{1}\right)$ are the following (see [8])

$$
\mathbf{y}\left(n j_{1}\right) \cdot \mathbf{I}=\mathbf{Y}\left(n j_{1}\right)
$$

or

$$
\mathbf{y}\left(n j_{1}\right)=\mathbf{Y}\left(n j_{1}\right)
$$

and

$$
\mathbf{y}\left(n j_{1}\right) \cdot\left[-\mathbf{E}\left(n j_{1}-2\right)\right]+\mathbf{a}\left(n j_{1}\right)=\mathbf{A}\left(n j_{1}\right)
$$

or

$$
\mathbf{a}\left(n j_{1}\right)=\mathbf{A}\left(n j_{1}\right)+\mathbf{y}\left(n j_{1}\right) \cdot \mathbf{E}\left(n j_{1}-2\right)
$$

and

$$
\mathbf{a}\left(n j_{1}\right) \cdot\left[-\mathbf{E}\left(n j_{1}-1\right)\right]+\mathbf{b}\left(n j_{1}\right)=\mathbf{B}\left(n j_{1}\right)
$$

or

$$
\mathbf{b}\left(n j_{1}\right)=\mathbf{B}\left(n \dot{j}_{1}\right)+\mathbf{a}\left(n j_{1}\right) \cdot \mathbf{E}\left(n j_{1}-1\right)
$$

and

$$
\mathbf{b}\left(n j_{1}\right) \cdot\left[-\mathbf{E}\left(n j_{1}\right)\right]=\mathbf{D}\left(n j_{1}\right)
$$

and

$$
\mathbf{b}\left(n j_{1}\right) \cdot\left[-\mathbf{x}\left(n j_{1}\right)\right]=\mathbf{X}\left(n j_{1}\right)
$$

and according to Eq. [10]

$$
\begin{aligned}
\mathbf{y} \cdot \mathbf{v}\left(n j_{1}-2\right)+\mathbf{a}\left(n j_{1}\right) \cdot \mathbf{v}\left(n j_{1}-1\right) & \\
& +\mathbf{b}\left(n j_{1}\right) \cdot \mathbf{v}\left(n j_{1}\right)=\mathbf{G}\left(n j_{1}\right)
\end{aligned}
$$

or

$$
\begin{aligned}
\mathbf{b}\left(n j_{1}\right) \cdot \mathbf{v}\left(n j_{1}\right)=\mathbf{G}\left(n j_{1}\right)-\mathbf{y}\left(n j_{1}\right) \\
\mathbf{v}\left(n j_{1}-2\right)-\mathbf{a}\left(n j_{1}\right) \cdot \mathbf{v}\left(n j_{1}-1\right)
\end{aligned}
$$

After $\mathbf{a}(j)$ is obtained by Eq. [30], $\mathbf{b}(j)$ can be determined directly by $\mathbf{E q}$. [32]. The values for $\mathbf{E}(j), \mathbf{x}(j)$, and $\mathbf{v}(j)$ are determined by solving Eq. [33], [34], and [36]. Note that Eq. [33], [34], and [36] have the same coefficient matrix $\mathbf{b}(j)$ so they can be solved by one call to a matrix-solver routine.

Calculation at the node $j=n j_{1}+1$ which follows the interior boundary condition will have the same form as that of $j=2$, i.e., Eq. [16], [18], and [20]. This applies for any node which follows a boundary point or an interface point.

When at the node $j=n j$, the calculation formulas are the same as that of $j=n j_{1}$ except that since the matrices $\mathbf{D}(n j)$ and $\mathbf{X}(n j)$ are null, it is meaningless to solve Eq. [33] and [34], so only Eq. [36] is used to solve for $\mathbf{v}(n j)$.
Once all of the elements of matrix $\mathbf{E}$ and vector $\mathbf{v}$ have been determined, Eq. [9] can be used to find the solution vectors $\mathbf{C}$ by backward substitution. The first equation is at $j=n j$

$$
\mathbf{I} \cdot \mathbf{C}(n j)=\mathbf{v}(n j)
$$

or simply

$$
\mathbf{C}(n j)=\mathbf{v}(n j)
$$

and the rest of the values of $\mathbf{C}$ can be calculated from this value and corresponding values of $\mathbf{E}$ and $\boldsymbol{v}$ by

$\mathbf{C}(j)=\mathbf{v}(j)+\mathbf{E}(j) \cdot \mathbf{C}(j+1)$

$$
\text { for }\left(2 \leq j \leq n j_{1}-1 \text { and } n j_{1}+1 \leq j \leq n j-1\right)
$$

At the interior and the left boundaries $\left(j=1\right.$ and $\left.n j_{1}\right)$, the following equation is used

$$
\mathbf{C}(j)=\mathbf{v}(j)+\mathbf{E}(j) \cdot \mathbf{C}(j+1)+\mathbf{x} \cdot \mathbf{C}(j+2)
$$

Although the above equations are based on an example with only one interior boundary point, MBAND can be applied to problems containing more than one interior boundary point.

\section{Example}

To illustrate the solution procedure, a simple set of coupled ordinary differential equations (11) is used. This set of equations describes the mole fractions of the gas and liquid reactants within a gas fed porous electrode of a fuel cell at steady state. Suppose the porous electrode consists of two layers of different materials, then the following set of equations applies to the two regions $[0,0.5]$ and $[0.5,1.0]$ in the domain $x$

$$
\begin{aligned}
& \frac{d^{2} y_{1}}{\partial x^{2}}-k_{1}^{\prime} y_{1}=0 \\
& \frac{d^{2} y_{2}}{\partial x^{2}}-k_{2}^{\prime} y_{1}=0
\end{aligned}
$$

with boundary conditions

at $x=0.0$

$$
\begin{aligned}
& y_{1}(0)=0.21 \\
& \left.\frac{d y_{2}}{d x}\right|_{x=0}=0
\end{aligned}
$$

at $x=0.5$

$$
\begin{aligned}
& \left.\frac{d y_{1}}{d x}\right|_{0.5-}=\left.\frac{d y_{1}}{d x}\right|_{0.5+} \\
& \left.\frac{d y_{2}}{d x}\right|_{0.5-}=\left.\frac{d y_{2}}{d x}\right|_{0.5+}
\end{aligned}
$$

at $x=1.0$

$$
\begin{aligned}
& \left.\frac{d y_{1}}{d x}\right|_{x=1}=0 \\
& y_{2}(1)=0.127
\end{aligned}
$$

This set of equations can be solved analytically

$$
\begin{gathered}
y_{1}=y_{1}(0)\left\{\frac{\cosh \left[k_{1}(1-x)\right]}{\cosh \left(k_{1}\right)}\right\} \\
y_{2}=y_{2}(1)+\frac{k_{2}^{2} y_{1}(0)}{k_{1}^{2} \cosh \left(k_{1}\right)}\left\{\cosh \left[k_{1}(1-x)\right]\right. \\
\left.-k_{1}(1-x) \sinh \left(k_{1}\right)-1\right\}
\end{gathered}
$$

where $k_{1}=\sqrt{k_{1}^{\prime}}$ and $k_{2}=\sqrt{k_{2}^{\prime}}$. 
The first step in solving Eq. [41]-[48] is to transform this set of ordinary differential equations into a system of algebraic equations by applying three-point finite difference approximations to each nodal point. These procedures as well as the determination of all the jacobian elements $\mathbf{A}, \mathbf{B}$, $\mathbf{D}, \mathbf{X}$, and $\mathbf{Y}$ are discussed in detail by Newman (6) and White (12). According to Eq. [45] and [46], the boundary conditions at the interior boundary $j=n j_{1}$ are

$$
\begin{gathered}
F_{1 \mathrm{j}}=\frac{3 C_{1, \mathrm{j}}-4 C_{1, \mathrm{j}-1}+C_{1, \mathrm{j}-2}}{2 \Delta x_{1}}-\frac{-3 C_{1, \mathrm{j}}+4 C_{1, \mathrm{j}+1}-C_{1, \mathrm{j}+2}}{2 \Delta x_{2}} \\
F_{2 \mathrm{j}}=\frac{3 C_{2, \mathrm{j}}-4 C_{2, \mathrm{j}-1}+C_{2, \mathrm{j}-2}}{2 \Delta x_{1}}-\frac{-3 C_{2, \mathrm{j}}+4 C_{2, \mathrm{j}+1}-C_{2, \mathrm{j}+2}}{2 \Delta x_{2}}
\end{gathered}
$$

where $C_{1, j}=y_{1}\left(x_{j}\right)$ and $C_{2, j}=y_{2}\left(x_{j}\right)$, and $\Delta x_{1}$ and $\Delta x_{2}$ are the grid sizes of regions 1 and 2 , respectively

$$
\Delta x_{1}=\frac{0.5}{n j_{1}-1} \quad \Delta x_{2}=\frac{0.5}{n j-n j_{1}}
$$

The elements of the coefficient matrices $\mathbf{A}, \mathbf{B}, \mathbf{D}, \mathbf{X}$, and $\mathbf{Y}$ at $j=n j_{1}$ are

$$
\begin{gathered}
\mathbf{Y}=\left[\begin{array}{cc}
\frac{1}{2 \Delta x_{1}} & 0 \\
0 & \frac{1}{2 \Delta x_{1}}
\end{array}\right] \\
\mathbf{A}=\left[\begin{array}{cc}
\frac{-4}{2 \Delta x_{1}} & 0 \\
0 & \frac{-4}{2 \Delta x_{1}}
\end{array}\right] \\
\frac{3}{2\left(\Delta x_{1}+\Delta x_{2}\right)} \\
0 \\
\mathbf{D}=\left[\begin{array}{cc}
\frac{-4}{2 \Delta x_{2}} & 0 \\
0 & \frac{-4}{2 \Delta x_{2}}
\end{array}\right] \\
\mathbf{x}=\left[\begin{array}{cc}
\frac{1}{2 \Delta x_{2}} & 0 \\
0 & \frac{1}{2 \Delta x_{2}}
\end{array}\right]
\end{gathered}
$$

Once expressions for all of the elements for each of the coefficient matrices are known at each node, a main program is used with MBAND to solve for $C_{\mathrm{ij}}$. The main program follows the same format as that required for $\operatorname{BAND}(J)$ routine (6). The calling sequence to MBAND is

\section{call mband(j, ibc, nbc, nj)}

where $\mathbf{j}$ is the current node, ibc is the boundary flag (ibc $=$ $\mathbf{i}$ at $\mathbf{i t h}$ boundary, $\mathbf{i b c}=0$ within regions), $\mathbf{n b c}$ is the number of boundaries (nbc $=$ number of regions +1 ), and $\mathbf{n j}$ is an integer array with a length of nbc which contains the number of nodes in each region. For example, for a tworegion problem with 11 nodes in the first region and 21 nodes in second region, the $\mathbf{n j}$ array (length of 3 ) has the values of 1,11 , and 31 , respectively.

The program was executed on a CRAY-YMP supercomputer, and Table I (please see p. 1688) lists the averaged relative errors ${ }^{1}$ of Eq. [41]-[48] solved using pentadia-

${ }^{1}$ Averaged relative error is defined as $\bar{e}=1 / n i 1 / n j \Sigma_{l}^{n i} \sum_{j}^{n j} \mid C(i, j)$ $-y_{i}\left(x_{j}\right) / C(i, j) \mid$, where $n i$ is the number of unknowns and $n j$ is the number of nodes.
Table III. CPU time statistics of the sparse matrix solver Y12MAF.

\begin{tabular}{lc}
\hline Module & CPU time $(\mathrm{ms})$ \\
\hline MAIN & $25(5.49 \%)$ \\
REORDER & $164(36.02 \%)$ \\
Y12MAF & $259(56.93 \%)$ \\
ZEROS & $7(1.49 \%)$ \\
TOTAL & 455
\end{tabular}

gonal BAND(J), a sparse matrix solver Y12MAF (13), and MBAND, respectively. It can be seen that the accuracies of all three methods are identical. Table II (please see p. 1688) presents the flowtrace statistics of the pentadiagonal BAND and MBAND subroutines after execution on the CRAY-YMP supercomputer (data in the parenthesis are percentages of total CPU time). It can be seen that when the pentadiagonal BAND is used, $82.19 \%$ of the total execution time is spent on BAND whereas MBAND consumes only $37.43 \%$ of the total CPU time. It is also worth noting that ten times more CPU time $(290 \mathrm{~ms} v s .29 \mathrm{~ms})$ was used with pentadiagonal BAND(J) compared to MBAND. A careful examination of the time consumed by the matrix solver LUDEC shows that when using pentadiagonal BAND $(J)$, the LUDEC module used $27 \mathrm{~ms}$, while when using MBAND, it took only $19 \mathrm{~ms}$. This is because in pentadiagonal BAND(J) the $\mathbf{D}$ and $\mathbf{X}$ arrays have dimensions of $n \times 5$, but in MBAND their dimensions are $n \times 3$. The time savings due to the column reduction of these two arrays is about $30 \%$. The contribution of the time reduction of LUDEC to the savings of total CPU time is significant, since this module is the most frequently called module by all of the BAND solvers. In terms of total CPU time used on the entire program, pentadiagonal BAND took $353 \mathrm{~ms}$, whereas MBAND used only $78 \mathrm{~ms}$, thereby resulting in about a fivefold speed increase.

A sparse matrix solver Y12MAF was also used to solve the same equations. According to the statistics shown in Table III, Y12MAF module took $259 \mathrm{~ms}$ and is much slower than MBAND.

Another important factor about using MBAND is its great savings of storage space. When the number of nodes is large, storage becomes a major problem for most of the matrix solvers including routines in IMSL and LINPACK, but not for MBAND.

\section{Acknowledgments}

The authors acknowledge gratefully that this work was supported by the Jet Propulsion Laboratory under contractor No. 958344 and by Cray Research, Incorporated. The authors would also like to thank G. Pillay for many helpful discussions.

Manuscript received Aug. 13, 1990; revised manuscript received Nov. 30, 1990.

Texas A\&M University assisted in meeting the publication costs of this article.

\section{REF'ERENCE'S}

1. T. V. Nguyen, R. E. White, and H. Gu, This Journal, 137,2998 (1990).

2. T. Yeu, T. V. Nguyen, and R. E. White, ibid., 135, 1971 (1988).

3. D. Bernardi and J. Newman, ibid., 134, 1309 (1987).

4. K. Tsaur and R. Pollard, ibid., 131, 975 (1984).

5. J. Newman, Ind. Eng. Chem. Fundam., 7, 514 (1968).

6. J. S. Newman, "Electrochemical Systems," Appendix C, Prentice-Hall, Inc., Englewood Cliffs, NJ (1991).

7. J. S. Newman, "Electrochemical Systems," PrenticeHall Inc., Englewood Cliffs, IJ (1991).

8. J. Van Zee, G. Kleine, R. E. White, and J. Newman, in "Electrochemical Cell Design," R. E. White, Editor, pp. 377-389, Plenum Press, Inc., New York (1984).

9. D. Fan and R. E. White, This Journal, 138, 17 (1991).

10. R. E. White, Ph.D. Thesis, University of California, Berkeley (1975).

11. R. E. White, M. A. Nicholson, L. G. Kleine, J. Van Zee, and R. Darby, This Journal, 131, 268 (1984).

12. R. E. White, Ind. Eng. Chem. Fundam., 17, 367 (1978).

13. Z. Zlatev, Technical Report No. 111, Department of Computer Science, University of Aarhus, Aarhus, Denmark (1980). 